\title{
Regulated Disorder: Posttranslational Modifications Control the RIN4 Plant Immune Signaling Hub
}

\author{
Tania Y. Toruño, ${ }^{1}$ Mingzhe Shen, ${ }^{2}$ Gitta Coaker, ${ }^{1}$ and David Mackey ${ }^{2,3,4, \dagger}$ \\ ${ }^{1}$ Department of Plant Pathology, University of California, Davis, CA 95616, U.S.A.; ${ }^{2}$ Department of Horticulture and Crop \\ Science, ${ }^{3}$ Department of Molecular Genetics, and ${ }^{4}$ Center for Applied Plant Sciences, The Ohio State University, Columbus, $\mathrm{OH}$ \\ 43210, U.S.A.
}

Accepted 16 October 2018.

\begin{abstract}
RIN4 is an intensively studied immune regulator in Arabidopsis and is involved in perception of microbial features outside and bacterial effectors inside plant cells. Furthermore, RIN4 is conserved in land plants and is targeted for posttranslational modifications by several virulence proteins from the bacterial pathogen Pseudomonas syringae. Despite the important roles of RIN4 in plant immune responses, its molecular function is not known. RIN4 is an intrinsically disordered protein (IDP), except at regions where pathogen-induced posttranslational modifications take place. IDP act as hubs for protein complex formation due to their ability to bind to multiple client proteins and, thus, are important players in signal transduction pathways. RIN4 is known to associate with multiple proteins involved in immunity, likely acting as an immune-signaling hub for the formation of distinct protein complexes. Genetically, RIN4 is a negative regulator of immunity, but diverse posttranslational modifications can either enhance its negative regulatory function or, on the contrary, render it a potent immune activator. In this review, we describe the structural domains of RIN4 proteins, their intrinsically disordered regions, posttranslational modifications, and highlight the implications that these features have on RIN4 function. In addition, we will discuss the potential role of plasma membrane subdomains in mediating RIN4 protein complex formations.
\end{abstract}

Plants rely on a series of passive and active defenses to protect against pathogens of different classes (Dodds and Rathjen 2010; Toruño et al. 2016). The waxy cuticle and cell walls provide physical barriers to pathogens. In addition, perception of conserved microbial features, such as bacterial flagellin or fungal chitin, as well as damage-associated components and other host-derived extracellular features by pattern recognition receptors (PRR) at the surface of plant cells activates a first layer of induced defense against diverse pathogens, referred to as PRR-triggered immunity (PTI). PTI induces formation of

Tania Y. Toruño and Mingzhe Shen contributed equally.

${ }^{\dagger}$ Corresponding author: D. Mackey; E-mail: mackey.86@osu.edu

Funding: All authors were supported by the National Institutes of Health (NIH) grant R01GM092772 awarded to G. Coaker and D. Mackey. Additional support for M. Shen and D. Mackey came from the Korean Rural Development Administration Next-Generation BioGreen 21 Program (System and Synthetic Agro-Biotech Center, PJ01326904).

(C) 2019 The American Phytopathological Society immune signaling complexes and cascades of defense responses, including activation of mitogen-activated protein kinases (MAPK), production of reactive oxygen species (ROS), induction of immune-related genes, and cell-wall fortification, among others (Zipfel 2014). Pathogens can overcome this first layer of immunity through secretion of pathogen effector proteins that disrupt defense responses (Toruño et al. 2016). However, plants have evolved immune receptors, called resistance $(\mathrm{R})$ proteins that recognize either effectors or effectorinduced perturbations of host cells (Chiang and Coaker 2015). The predominant class of $\mathrm{R}$ proteins is intracellular nucleotidebinding leucine-rich repeat receptors (NLR), such as resistance to Pseudomonas syringae pv. maculicola 1 (RPM1), that respond to effectors by eliciting a frequently robust immune response referred to as NLR-triggered immunity (NTI) (Dodds and Rathjen 2010). There is significant overlap in immune signaling mediated by PRR and NLR (Thomma et al. 2011).

RPM1-interacting protein 4 (RIN4) is conserved in land plants and functions at the intersection of PTI, effector-mediated immune suppression, and NTI. Genetically, RIN4 negatively regulates PTI as plants lacking or overexpressing RIN4 display enhanced or reduced defense responses, respectively (Kim et al. 2005b). RIN4 is also subjected to diverse posttranslational modifications (PTM) that regulate immune signaling in varied manners. Multiple pathogen effectors induce distinct RIN4 PTM, including cleavage, phosphorylation, and acetylation, that lead to inhibited immune defense (Afzal et al. 2011; Axtell and Staskawicz 2003; Chung et al. 2011, 2014; Kim et al. 2005a; Lee et al. 2015b; Lewis et al. 2011; Liu et al. 2011; Mackey et al. 2002, 2003) (Fig. 1). However, multiple, independently evolved NLR elicit NTI in response to effector-induced modification of RIN4 (Axtell and Staskawicz 2003; Mackey et al. 2002; 2003). The conservation of RIN4 across land plants, its importance in regulating both branches of plant immunity, and its targeting by multiple pathogen effectors highlight RIN4 as a key hub of the plant immune system. In this review, we will discuss the localization, structure, PTM, and associated proteins of RIN4 from several plant species, with an emphasis on Arabidopsis thaliana RIN4 (AtRIN4, hereafter RIN4). The consequences of these features of RIN4 structure and function on immunity are described.

\section{RIN4 LOCALIZATION AND STRUCTURE}

RIN4 is peripherally tethered to the plasma membrane (PM) through posttranslational modification of a cysteine-rich membrane-anchoring motif at its C-terminus (Chisholm et al. 2005; Kim et al. 2005a; Takemoto and Jones 2005). One or 
more sulfhydryl groups of cysteine residues (C203 to C205) is modified by S-acylation (also known as palmitoylation), which is the reversible addition of saturated fatty acids, usually palmitate or stearate (Hemsley 2015). Fatty acyl transferases, which catalyze $\mathrm{S}$-acylation, comprise large protein families in plants, including 24 in Arabidopsis (Batistic 2012). While the specific fatty acyl transferase or transferases that modify RIN4 remain unknown, the resulting membrane tethering is likely a generalized feature, since the majority of RIN4 homologs possess a C-terminal cysteine-rich membrane-anchoring motif (Afzal et al. 2013; Sun et al. 2014) and PM localization has been observed for RIN4 homologs in soybean (GmRIN4) (Selote and Kachroo 2010) (Fig. 1).

Although RIN4 is a key regulator of immunity and a target of several pathogen effectors, its molecular function remains an enigma. RIN4 is not known to possess enzymatic activity and lacks similarity to proteins of known function. However, RIN4 is an intrinsically disordered protein (IDP) based on in silica predictions and circular dichroism spectroscopy (Lee et al. 2015a; Sun et al. 2014). Typically, IDP are enriched in charged, hydrophilic amino acids and depleted in bulky, hydrophobic amino acids (Wright and Dyson 2015). Disordered regions within IDP, which lack secondary or tertiary structure but can transition to a more ordered or folded state upon client binding and through association with multiple binding partners, often carry out important cellular functions (Oldfield and Dunker 2014). Intrinsically disordered regions can bind partner proteins through the presence of relatively short rigid segments, called molecular recognition features (MoRF), that are present within long disordered regions (Mohan et al. 2006; Oldfield et al. 2005b). Keys to the role of IDP in signaling are their rapid and specific association with multiple signaling partners, the low affinity of those interactions that facilitate termination of the signaling complex, and the modulation of association and dissociation events by PTM of highly accessible sites (Oldfield and Dunker 2014).

The binding promiscuity of IDP allow them to regulate many signal transduction pathways and integrate multiple environmental signals. For example, GRAS (for GAI, RGA, SCR) transcriptional regulators depend on their N-terminal intrinsic disordered regions to bind to multiple partners (Sun et al. 2012). DELLA proteins, a subfamily of the GRAS protein family, are key regulators of gibberellin, jasmonate, and light signaling (Sun et al. 2012). DELLAs regulate diverse signaling processes through dynamic interactions of their intrinsically disordered regions with hormone receptors and light-responsive transcription factors (de Lucas et al. 2008; Feng et al. 2008; Hou et al. 2010; Sun 2011; Wild et al. 2012). The Arabidopsis bZIP transcription factor HY5 (for elongated hypocotyl 5) and the blue light photoreceptor CRY1 (for cryptochrome 1) are also well-studied plant signaling proteins possessing long disordered regions (Partch et al. 2005; Sun et al. 2013; Yoon et al. 2006). A disorder prediction study revealed that $23 \%$ of Arabidopsis proteins are mostly disordered (Oldfield et al. 2005a). Similarly, approximately $66 \%$ of eukaryotic signaling proteins possess long predicted stretches of intrinsic disorder (Hsu et al. 2013; Iakoucheva et al. 2002). Therefore, regulation of individual and integration of multiple signaling pathways by IDP may be a common feature in plants.

RIN4 homologs from numerous plant species are predicted to be intrinsically disordered (Sun et al. 2014) (Fig. 1). RIN4 possesses two plant-specific nitrate-induced (NOI) domains (Kim et al. 2005a). NOI domains were identified in a screen for nitrate-induced genes but, so far, there is no evidence for a role of NOI proteins in nitrogen metabolism. Proteins possessing either one or two NOI domains containing the conserved PxFGxW and Y/FTxxF motifs and a C-terminal membraneanchoring motif are present across land plants (Afzal et al.
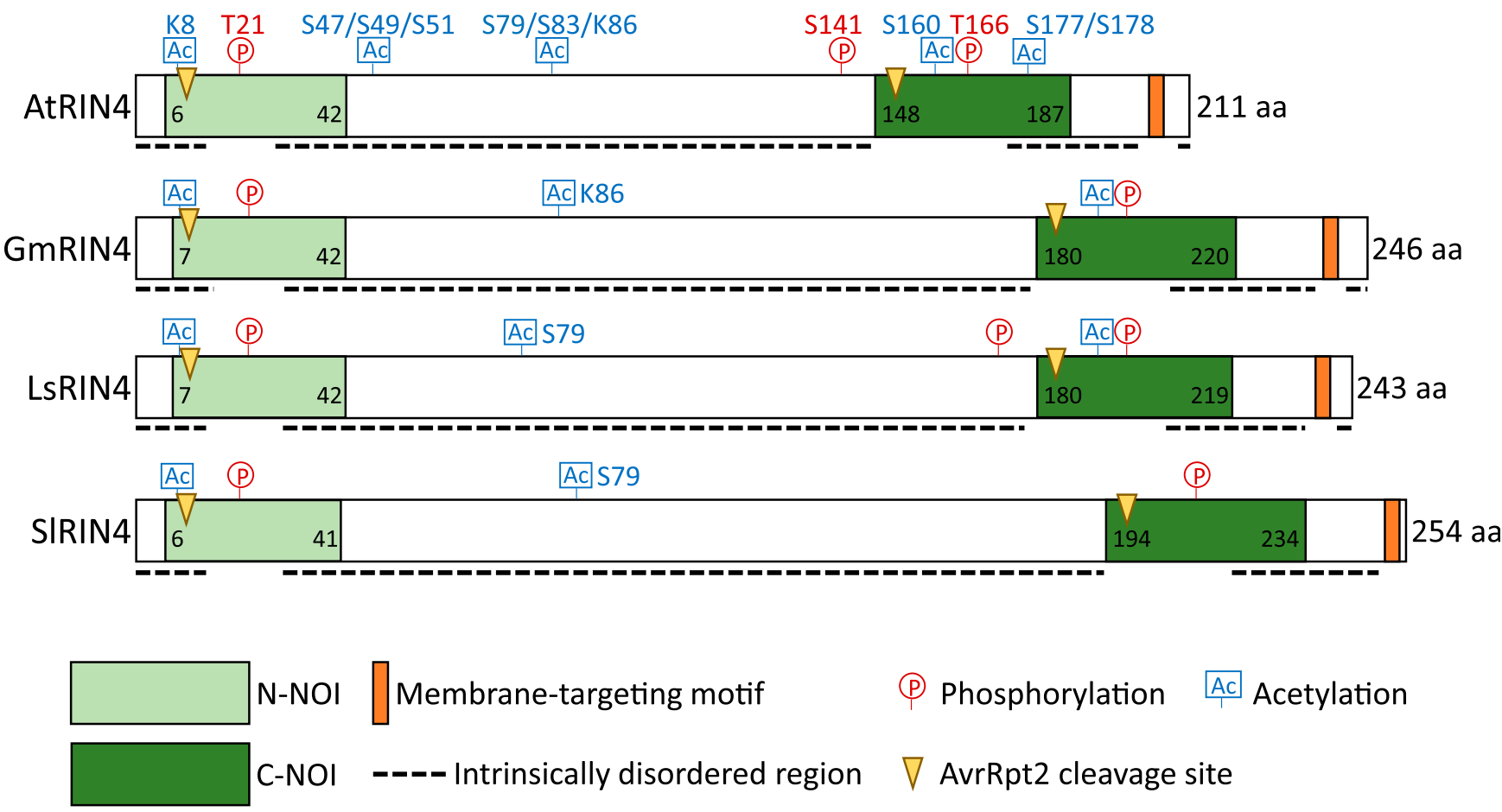

(P) Phosphorylation Acetylation

\section{AvrRpt2 cleavage site}

Fig. 1. RIN4 proteins, their domain architecture, sites of posttranslational modification, and regions of intrinsic disorder. Schematic diagram of Arabidopsis RIN4 (AtRIN4), soybean RIN4 (GmRIN4a), lettuce RIN4 (LsRIN4), and tomato RIN4 (SIRIN4) proteins. Positions of N-terminal NOI, C-terminal NOI, and membrane-anchoring domains are indicated as light green, dark green and orange blocks, respectively. Indicated are phosphorylated and acetylated residues and AvrRpt2-cleavage sites of AtRIN4 and their conservation in other RIN4 homologs. Intrinsically disordered regions indicated for each RIN4 homolog were predicted, using the PrDOS (protein disorder prediction system) server. 
2013). A combination of bioinformatic and experimental approaches has demonstrated that RIN4 possesses multiple MoRF and long disordered regions (Lee et al. 2015a; Sun et al. 2014). Interestingly, the NOI domains and the C-terminal membraneanchoring motif are the only regions of RIN4 predicted to be ordered (Lee et al. 2015a) (Fig. 1). Though RIN4 homologs share little amino acid sequence similarity outside of their ordered regions (Afzal et al. 2013), the conservation of regions of similar size with predicted disorder indicates a functional role for these regions. We propose that RIN4 is a key signaling hub at the PM that can help coordinate protein complex formation and immune signaling, given its large number of associated proteins and IDP structure.

\section{POSTTRANSLATIONAL MODIFICATIONS REGULATE RIN4 FUNCTION}

RIN4 is subjected to multiple types of PTM induced by both virulence factors of potential pathogens and host enzymes. To survive and proliferate in host cells, most gram-negative bacterial pathogens use the type III secretion system to directly deliver a number of virulence proteins, called type III effectors (T3E), into host cells. T3E manipulate host targets to modulate host physiology and defense and thus create an advantageous environment for the bacteria (Cunnac et al. 2009; Deslandes and Rivas 2012; Jones and Dangl 2006; Khan et al. 2016). Many T3E contain catalytic domains with enzymatic activities capable of modifying plant proteins or co-opt host enzymes to modify other host targets (Block et al. 2008; Toruño et al. 2016). The Pseudomonas syringae T3E AvrB, AvrRpm1, AvrRpt2, HopF2, and HopZ3 have been demonstrated to directly target RIN4 (Axtell and Staskawicz 2003; Lee et al. 2015b; Luo et al. 2009; Mackey et al. 2002, 2003; Wilton et al. 2010). Further indication that RIN4 functions as a hub in plant innate immunity comes from the observation that, in addition to P. syringae T3E (Axtell and Staskawicz 2003; Luo et al. 2009; Mackey et al. 2002, 2003; Wilton et al. 2010), RIN4 is also targeted for various PTM by host proteins (Chung et al. 2014; Kaundal et al. 2017; Li et al. 2014a; Liu et al. 2011; Luo et al. 2009). In the following paragraphs, we discuss PTM of RIN4 and the implications of those modifications with respect to PTI and NTI.

\section{Phosphorylation.}

Phosphorylation of multiple residues of RIN4 (Fig. 1) has been implicated in its regulation of plant immunity (Fig. 2). AvrRpm1 and AvrB are two sequence-unrelated T3E that share in common their ability to activate the Arabidopsis NLR RPM1 (Bisgrove et al. 1994; Grant et al. 1995). AvrRpm1 and AvrB each possess a short $\mathrm{N}$-terminal sequence including a G2 myristoylation site and a C3 palmitoylation site. The G2 residue is critical for accumulation and membrane localization of AvrRpm1 and AvrB inside plant cells (Nimchuk et al. 2000). Both AvrRpm1 and AvrB coimmunoprecipitate with RIN4, and AvrB induces phosphorylation of an evolutionarily conserved threonine residue at position 166 of RIN4 (Chung et al. 2011; Liu et al. 2011; Mackey et al. 2002) (Fig. 1). Both AvrB and AvrRpm1 induce accumulation of a plant receptorlike cytoplasmic kinase (RLCK) called RIPK (RPM1-induced protein kinase) (Liu et al. 2011). RIPK directly phosphorylates RIN4 at T166. A ripk mutant shows reduced but not abolished phosphorylation of RIN4 in response to AvrB and to a lesser extent AvrRpm1 (Liu et al. 2011). Recently, additional members of the RLCK subfamily have been demonstrated to phosphorylate RIN4 T166, and higher order RLCK mutants are more strongly inhibited in RPM1 activation, indicating redundancy in RLCK-mediated RIN4 phosphorylation (Xu et al. 2017).

Phosphorylation of RIN4 T166 plays contrasting roles in its ability to suppress PTI and activate NTI. NLR are posited to respond to host perturbations associated with the virulence activity of pathogen effectors (Jones and Dangl 2006). Consistent with this hypothesis, phosphorylation of RIN4 T166 is directly linked to the activation of NTI by RPM1 (Fig. 2).
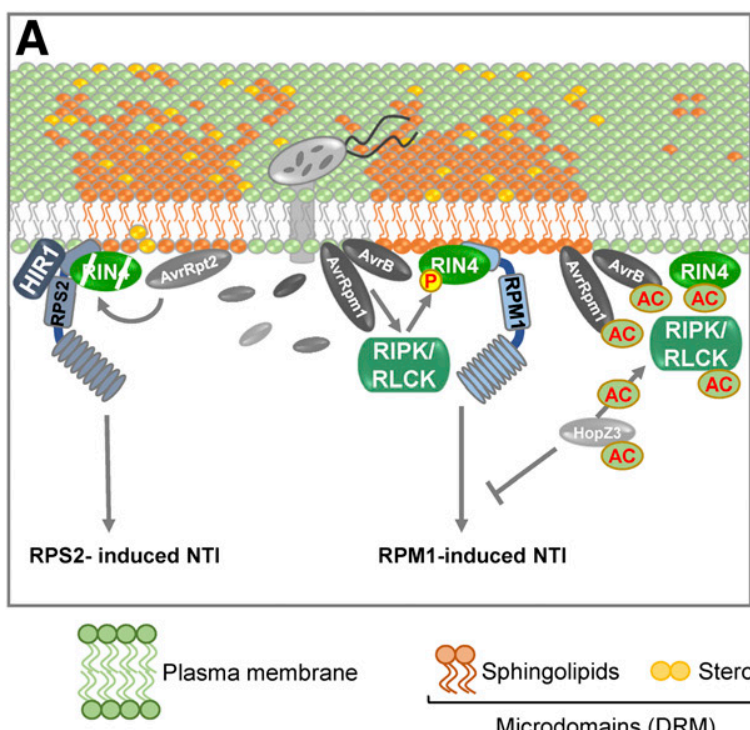

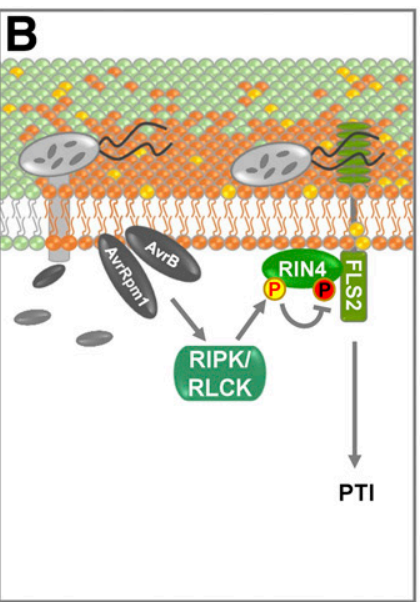

(P) T166 phosphorylation

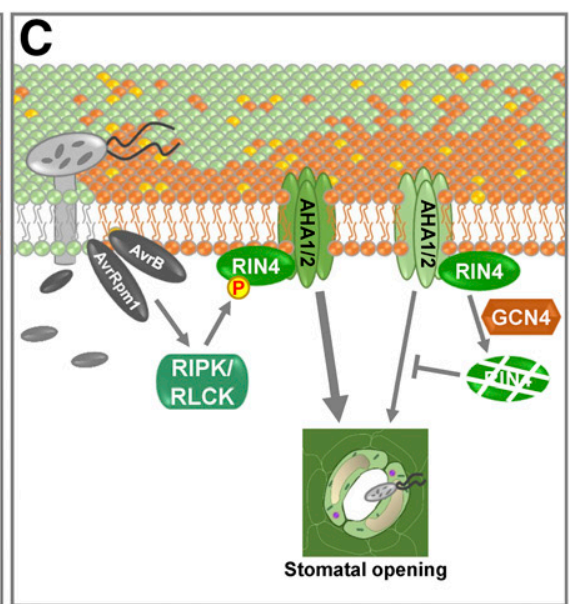

S141 phosphorylation
Acetylation

Fig. 2. RIN4-associated proteins are enriched in detergent-resistant membranes (DRM), which are heterologous regions rich in sphingolipids and sterols and may constitute plasma membrane microdomains that serve as platforms for protein complex formation. A, Type III effectors from Pseudomonas syringae trigger nucleotide-binding leucine-rich repeat receptor (NLR)-triggered immunity (NTI) mediated by the RPS2 or RPM1 NLR through AvrRpt2-induced proteolysis of RIN4 or AvrB/AvrRpm1-induced phosphorylation of RIN4 T166, respectively. RIN4 is phosphorylated by RIPK and additional receptor-like cytoplasmic kinases (RLCK). The HopZ3 effector is an acetyltransferase that acetylates the RIN4 complex to inhibit NTI triggered by RPM1. B, Effectors targeting RIN4 suppress PTI promoted by phosphorylation of S141 through AvrB/AvrRpm1-induced phosphorylation of T166. C, The contribution of RIN4 to activity of plasma membrane $\mathrm{H}^{+}$-ATPases, which promote stomatal opening, can be enhanced by AvrB/AvrRpm1-induced phosphorylation of T166 or inhibited by ubiquitin-dependent degradation of RIN4 induced by general control non-repressible-4 (GCN4). 
Phosphomimetic mutants T166D and T166E are sufficient for T3E-independent RPM1 activation upon transient expression in Nicotiana benthamiana or stable expression in Arabidopsis (Chung et al. 2011; Liu et al. 2011). Conversely, the phosphonull mutant T166A displays compromised RPM1 activation in response to AvrB (Chung et al. 2011; Lee et al. 2015a; Liu et al. 2011). The cyclophilin ROC1 (for rotamase CYP1) also associates with RIN4 and enforces T166 phosphorylation to enhance RPM1-mediated responses (Li et al. 2014a). In Arabidopsis genotypes lacking NLR that respond to perturbations of RIN4, complementation with phosphomimetic RIN4 T166D/E suppresses PTI responses (Chung et al. 2014; Lee et al. 2015a). RIN4 T166 phosphomimetic complementation lines exhibit enhanced growth of $P$. syringae and reduced microbe-associated molecular pattern (MAMP)-induced ROS burst (Chung et al. 2014; Lee et al. 2015a). Thus, AvrB co-opts host RLCK to promote virulence via phosphorylation of RIN4 T166 and plants with RPM1 respond to this perturbation by eliciting NTI (Fig. 2).

RIN4 phosphorylation at specific residues is differentially involved in activation or suppression of PTI signaling, indicating that phosphorylation is a molecular switch for RIN4 function (Chung et al. 2011, 2014; Lee et al. 2015a). A 22amino acid peptide MAMP from the flagellin protein (flg22) triggers phosphorylation of RIN4 at serine 141 by PTIregulatory host RLCK, including Botrytis-induced kinase1 (BIK1) and PBS1-like 1 (Chung et al. 2014; Li et al. 2014b; Lu et al. 2010; Zhang et al. 2010). Phosphorylation of S141 enhances PTI signaling; Arabidopsis complementation lines expressing phospho-null RIN4 S141A exhibit reduced callose deposition and bacterial growth inhibition after flg22 treatment, with the S141E phosphomimetic mutant exhibiting an opposite phenotype (Chung et al. 2014). Secretion of AvrB into plant cells, which is subsequent to MAMP-induced phosphorylation, counteracts the effect of S141 phosphorylation by inducing phosphorylation of T166, which enhances RIN4-mediated repression of PTI (Chung et al. 2014; Lee et al. 2015a). Because phosphorylation of T166 is epistatic to S141, AvrB effectively antagonizes PTI signaling by restoring the repressive function of RIN4 (Chung et al. 2014) (Fig. 2). The S141 and T166 residues of RIN4 are widely conserved in homologs from other plants, indicating that these counteracting phosphorylation events are a conserved feature of immune regulation in plants (Fig. 1). Given that each of these sites is phosphorylated by distinct host RLCK, it is likely this mode of regulation was co-opted by effectors for pathogen benefit.

In addition to heightening plant defense against pathogens that have invaded plant intracellular spaces, PTI can also induce pre-invasion defense by promoting closure of stomatal openings (Melotto et al. 2006). RIN4, including its phosphorylation status, plays an important role in regulation of stomatal opening (Lee et al. 2015a; Liu et al. 2009). AHAs are PM H ${ }^{+}$-ATPases that regulate stomatal movement (Elmore and Coaker 2011). Activation of AHA induces stomatal opening to facilitate bacterial pathogen invasion (Assmann et al. 1985; Elmore and Coaker 2011; Kim et al. 2010; Shimazaki et al. 1986). RIN4 interacts with AHA1 and AHA2 in yeast, in vitro and in planta (Fig. 2). Overexpression of RIN4 or a rin4 mutant enhances

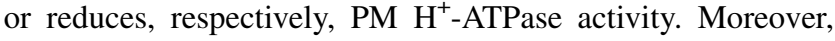
surface-inoculated $P$. syringae fails to reopen stomata in the rin4 mutant (Liu et al. 2009). Notably, increased ability of RIN4 to activate PM $\mathrm{H}^{+}$-ATPase activity and, thus, suppress pre-invasion defense was associated with phosphorylation of T166 (Lee et al. 2015a). RIN4 also associates with GCN4 (general control nonrepressible 4), an $\mathrm{AAA}^{+}$-ATPase family protein that functions in stomatal movement during biotic and abiotic stress (Kaundal et al. 2017). Studies of GCN4 further link RIN4 to stomatal function during plant immunity. GCN4 interacts with RIN4 and, by promoting proteasome-mediated degradation of RIN4, reduces $\mathrm{H}^{+}$-ATPase activity and stomatal closure (Kaundal et al. 2017) (Fig. 2). Taken together, these results indicate that RIN4 PTM and interaction with GCN4 regulate the function of $\mathrm{PM} \mathrm{H}^{+}$-ATPases involved in stomatal defense.

\section{Proteolysis.}

Another T3E that targets RIN4 is the cysteine protease AvrRpt2. Upon delivery into plant cells, AvrRpt 2 is activated by auto-cleavage dependent on a host cyclophilin (Coaker et al. 2005). The activated form of AvrRpt 2 associates with the PM, likely through a myristoylation site at the newly created $\mathrm{N}$ terminus, where it directly cleaves RIN4 at two sites present in the N- and C-terminal NOI domains (Axtell et al. 2003; Chisholm et al. 2005; Jin et al. 2003; Kim et al. 2005a; Takemoto and Jones 2005) (Figs. 1 and 2). Cleavage of AvrRpt2 eliminates full-length RIN4 and produces two major, short-lived products, ACP2 (AvrRpt2-cleavage product 2) and ACP3 (Afzal et al. 2011). ACP2 (amino acids 11 to 152) is an internal, soluble fragment of RIN4 that includes the majority of the cleaved N-terminal NOI domain. ACP3 (amino acids 153 to 211) is the C-terminal, membrane-tethered fragment of RIN4 that includes the majority of the cleaved $\mathrm{C}$-terminal NOI domain.

Plants lacking RIN4 show enhanced PTI responses (Kim et al. 2005b; Liu et al. 2009). Thus, the relationship between cleavage of RIN4 and the virulence activity of AvrRpt 2 was initially unclear. Indeed, AvrRpt 2 does have virulence activity independent of RIN4 targeting (Lim and Kunkel 2004). However, AvrRpt 2 also targets RIN4 to produce cleavage products that are suppressors of PTI. ACP2 prevents callose deposition after flg22 treatment and both ACP2 and ACP3 promote the growth of a type III secretion-deficient mutant of $P$. syringae. Notably, though short-lived, ACP2 and ACP3 are hyperactive suppressors of PTI, relative to intact RIN4 (Afzal et al. 2011). Within RIN4, both the N- and C-terminal NOI domains contribute to its negative regulation of PTI (Afzal et al. 2011), indicating that the NOI fragments contained in soluble ACP2 and membrane-tethered ACP3 retain activity following cleavage by AvrRpt2. The effect of ACP2 and ACP3 on PM $\mathrm{H}^{+}$-ATPase activity is not known.

Just as RPM1 responds to AvrB- and AvrRpm1-induced perturbations of RIN4, another Arabidopsis NLR, RPS2 (resistance to $P$. syringae 2), responds to AvrRpt2-induced cleavage of RIN4 (Fig. 2). Plants lacking RIN4 display seedling lethality, due to ectopic activation of RPS2 (Mackey et al. 2003). Based on this observation and the activation of RPS2 upon RIN4 cleavage in $N$. benthamiana (Day et al. 2005), it has been widely proposed that the activation of RPS2 results from the disappearance of RIN4. However, requirement for the nonrace-specific disease resistance 1 (NDR1) defense regulatory protein highlights an intriguing difference between ectopic activation of RPS2 in the absence of RIN4 and effector-induced activation of RPS2 by AvrRpt2. Specifically, the ectopic activation of RPS2 is independent of the NDR1, while RPS2dependent NTI triggered by AvrRpt 2 is absolutely dependent on NDR1 (Aarts et al. 1998; Belkhadir et al. 2004). Notably, the contribution of NDR1 to AvrRpt2-induced activation of RPS2 requires its interaction with RIN4 (Day et al. 2006). The role of ACP2, ACP3, and NDR1 for the activation of RPS2 during Pseudomonas infection remains to be demonstrated.

\section{ADP-ribosylation.}

The addition of ADP-ribose moieties to proteins is a reversible PTM that regulates a variety of cellular processes (Palazzo 
et al. 2017). The T3E HopF2 is an ADP-ribosyltransferase that contains an N-terminal G2 myristoylation site required for its localization to the PM (Robert-Seilaniantz et al. 2006; Wang et al. 2010; Wilton et al. 2010). HopF2 directly interacts with and ADP-ribosylates RIN4 (Wang et al. 2010; Wilton et al. 2010). Targeting of RIN4 by HopF2 contributes to the suppression of both NTI and non-NTI host defenses (Wilton et al. 2010). The ability of HopF2 to promote the growth of a virulent strain of $P$. syringae (non-NTI) is abolished in rin4 mutant plants. Also, the action of HopF2 suppresses AvrRpt2-induced cleavage of RIN4, thereby inhibiting RPS2-dependent NTI. Taken together, these results demonstrate that RIN4 is a major virulence target of HopF2. Interestingly, AvrRpm1, which influences RIN4 phosphorylation, also contains a fold homologous to ADP-ribosyltransferases (Cherkis et al. 2012). It will prove interesting to determine if, like its inhibition of proteolysis, ADPribosylation of RIN4 also alters its phosphorylation by host RLCK.

\section{Acetylation.}

Acetylation is a reversible PTM perhaps best known as a histone mark that regulates transcription (Drazic et al. 2016). RIN4 is targeted by the cytoplasmic T3E HopZ3, a member of the YopJ family of acetyltransferases (Lee et al. 2015b; Lewis et al. 2011). While lysine is a commonly acetylated residue, YopJ from Yersinia pestis targets serine and threonine residues in the activation loop of a MAPK kinase, thereby blocking phosphorylation and kinase activation (Mukherjee et al. 2006). HopZ3 suppresses RPM1-dependent defenses by acetylating serine, threonine, lysine, and histidine residues of multiple members of the RPM1 immune complex, including RPM1, RIPK, and RIN4, as well as an RPM1-activating T3E AvrRpm1 or AvrB (Lee et al. 2015b). HopZ3, which directly interacts with RIN4 and RIPK, reduces phosphorylation of RIN4 following bacterial infection. Possibly explaining this effect, invitro acetylation of RIPK by HopZ3 inhibited its kinase activity. Therefore, HopZ3 interferes with RPM1-dependent immune responses via acetylation of the immune complex, including RIN4 (Fig. 1, 2). The effect of HopZ3-induced acetylation on the function of RIN4 during PTI remains to be determined.

\section{RIN4 HOMOLOGS IN OTHER PLANT SPECIES}

RIN4 is a member of a highly conserved protein family found in monocots and dicots including tomato, soybean, common bean, lettuce, apple, corn, and rice, as well as more ancient plants such as moss (Afzal et al. 2013). RIN4 homologs from diverse plant species typically contain NOI domains with motifs capable of being cleaved by AvrRpt 2 and a C-terminal membrane-anchoring motif (Fig. 1). Studies in various plant species have extended our understanding of RIN4 function.

\section{Soybean.}

Soybean has undergone genome duplication events and haploid genome analyses indicate it is a partially diploidized tetraploid (Shultz et al. 2006). Soybean contains at least four RIN4 homologs (GmRIN4a to GmRIN4d), each containing the hallmark Arabidopsis RIN4 motifs (Fig. 1). GmRIN4a and GmRIN4b are most similar to RIN4, sharing approximately $50 \%$ identity. GmRIN4b but not GmRIN4a could restore RPM1 function when introduced into rin4 mutant Arabidopsis plants (Selote and Kachroo 2010). The soybean NLR RPG1-B (resistance to $P$. syringae pv. glycinea), which elicits NTI in response to AvrB, requires GmRIN4a and GmRIN4b for its function (Ashfield et al. 1995; Selote et al. 2013). AvrB induces phosphorylation of GmRIN4b on residues analogous to RIN4
T21 and T166 and those phosphorylation events prevent the interaction of GmRIN4b with both GmRIN4a and RPG1-B (Selote et al. 2013). In N. benthamiana, mimicking phosphorylation of GmRIN4b is sufficient to activate RPG1-B (Selote et al. 2013). Unlike RPM1, which has dual specificity for both AvrB and AvrRpm1, soybean possesses a distinct NLR, RPG1-R, which specifically detects AvrRpm1 (Ashfield et al. 1995). Rpg 1 b and Rpg $1 r$ are evolutionarily closely related but are unrelated to RPMI (Ashfield et al. 2014). AvrRpt 2 cleaves all four soybean RIN4 homologs and prevents the AvrB- or AvrRpm1-dependent activation of RPG1-B, RPG1-R, or RPM1 (Ashfield et al. 2004; Ritter and Dangl 1996). These data highlight the targeting of RIN4 by multiple effectors in diverse plants and the convergent evolution of NLR responsive to T3Einduced perturbation of RIN4 and its homologs.

\section{Lettuce.}

Necrotic responses in plant hybrids often result from autoimmune responses and inappropriate NLR pairing (Bomblies et al. 2007). The presence of RIN4 can regulate autoimmunity in lettuce, indicating that RIN4 may be monitored by lettuce NLR. The case of necrosis in an interspecific hybrid of lettuce (Lactuca sativa and Lactuca saligna) results from an interaction between two genetic loci, one of which encodes for lettuce RIN4 (LsRIN4 [Fig. 1]), which shares NOI domains, a C-terminal membrane-anchoring motif, and 54\% identity with RIN4 (Jeuken et al. 2009). Hybrid necrosis was due to three polymorphisms between the two Lactuca species, two residues were located outside NOI domains and one was in close proximity to the C-terminal membrane-anchoring motif (Jeuken et al. 2009). Notably, the RIN4-mediated response in lettuce is associated with resistance to downy mildew of lettuce. Thus, the interaction of RIN4 and other loci, including NLR, will be essential to breeding efforts in lettuce and likely other plant species.

Tomato.

RIN4 is directly subjected to proteolysis by the AvrRpt2 effector as well as a host protease. The tomato homolog of RIN4, SIRIN4 (Fig. 1), is proteolyzed upon activation of Prf (Luo et al. 2009). Prf is a tomato NLR that recognizes perturbation of the host RLCK Pto by the AvrPto effector (Pedley and Martin 2003). Activation of Prf, either by AvrPto or by an autoactive variant of Pto, induces proteolysis of SIRIN4. Like RIN4, SIRIN4 contains two NOI domains, each with an AvrRpt2 cleavage site (Chisholm et al. 2005; Luo et al. 2009). Remarkably, cleavage of SIRIN4 that follows activation of Prf occurs at the precise sites cleaved by AvrRpt2, which indicates that tomato and $N$. benthamiana encode a protease with specificity matching AvrRpt2. Thus, targeting of NOI domaincontaining proteins by AvrRpt 2 likely impinges upon a mechanism of action utilized by plants in other contexts, including activation of Prf. Indeed, reducing the levels of SIRIN4 in tomato compromised Prf-mediated resistance in response to AvrPto (Luo et al. 2009). Perhaps in a related manner, GmRIN4c and GmRIN4d are required for the activity of NLR other than RPG1-B and RPG1-R in soybean (Selote et al. 2013). Also consistent with the notion that a plant endogenous pathway leads to proteolysis of RIN4, expressing either of two other P. syringae T3E, HopAM1 or HopQ1-1, elicits RIN4 cleavage in $N$. benthamiana at the same sites as targeted by AvrRpt2 (Luo et al. 2009). Thus, proteolysis of RIN4 at specific sites by host proteases is likely a mechanism of immune regulation that was co-opted by AvrRpt2. Identification of the plant protease that targets this site would likely provide significant insight into the function of RIN4 and other NOI domain-containing proteins in plants. 
Rice.

Pii-2 is an NLR that triggers disease resistance to the rice blast fungus Magnaporthe oryzae upon recognizing the effector protein AVR-Pii (Fujisaki et al. 2015). Interestingly, Pii-2 has an integrated C-terminal domain containing the NOI/RIN4 core motif (PxFGxW), which is the site cleaved by AvrRpt2 (Fujisaki et al. 2015). Pii-2 does not directly interact with AVRPii. Rather, the integrated NOI core motif directly interacts with OsExo70-F3, which is a component of the exocyst complex and, presumably, either a virulence or decoy target of AVR-Pii. Thus, Pii-2 activation results from indirect perception of AVRPii via its targeting of OsExo70-F3 (Fujisaki et al. 2015). The association of Exo70 proteins with proteins containing NOI domains is likely generalizable, given the recent evidence that RIN4 interacts with AtExo70B1 (Sabol et al. 2017). Given that the NOI-Exo70 interaction was co-opted into an NLR integrated domain indicates that the interaction of RIN4 with Exo70 proteins is an ancient function of RIN4 that has been targeted by pathogenic effectors. Though the effectors currently known to target RIN4 are bacterial T3E, the findings in rice that a fungal effector from $M$. oryzae targets the interaction of an EXO70 protein with a conserved motif from RIN4 indicates that effectors from other classes of plant pathogens likely target RIN4.

\section{MEMBRANE MICRODOMAINS AS PLATFORMS FOR RIN4 COMPLEX FORMATION}

The plant PM is an important compartment in which essential biological processes take place, including protein trafficking to the inside and outside of the cell (endocytosis and exocytosis), metabolite and hormone transport, perception of microbes, and the subsequent activation of immune signaling events. RIN4 as well as many of its validated, associated proteins localize to the PM (Fig. 2). Targeting of RIN4-associated proteins to the PM occurs via different mechanisms. Some of these include the presence of transmembrane domains (NDR1, FLS2), addition of fatty acids in the form of $\mathrm{N}$-myristoylation and $\mathrm{S}$-acylation posttranslational modifications (RIPK), and attachment of GPI anchors (NDR1) (Coppinger et al. 2004; Day et al. 2006; Liu et al. 2011; Knepper et al. 2011; Robatzek et al. 2006). Two NLR known to interact with RIN4, RPM1 and RPS2, do not contain predicted transmembrane domains or membrane-targeting motifs but, nonetheless, localize to the PM. RPM1 is a peripheral PM protein based on its solubilization by chemical treatments (Boyes et al. 1998). Unlike RPM1, RPS2 is an integral membrane protein, since treatments that solubilize peripheral membrane proteins failed to release RPS2 from the membrane (Axtell and Staskawicz 2003). In addition to host-associated proteins, $P$. syringae effectors targeting RIN4 also localize to the PM. AvrRpm1 and AvrB carry myristoylation sites that are acylated upon delivery into plant cells, mediating their PM localization (Nimchuk et al. 2000; Shan et al. 2000). Also, the enzymatically active fragment of AvRpt 2 contains a putative $\mathrm{N}$-terminal myristoylation site that is responsible for its PM localization (Jin et al. 2003). These data indicate that the function of RIN4 as a hub for plant immunity occurs at the PM, which provides a platform for RIN4 protein complex formation (Fig. 2).

The plant PM, which consists of a heterogeneous layer of proteins and lipids, dynamically reconfigures in response to physiological and environmental cues. In addition to endo- and exocytic protein trafficking, these reconfigurations also include the assembly of protein complexes within discrete membrane microdomains. Such membrane microdomains are liquidordered phase domains rich in sphingolipids and sterols that, due to resistance to solubilization by nonionic detergents, are often a constituent within preparations of detergent-resistant membranes (DRM) (Malinsky et al. 2013). Membrane microdomains have been proposed to act as organizing platforms that facilitate protein-protein and protein-lipid interactions and, thus, cellular signaling, transport, and membrane trafficking (Malinsky et al. 2013; Qi and Katagiri 2012). The formation of membrane microdomains provides distinct spatial clustering of PM-localized receptors, including the bacterial flagellin receptor FLS2, the respiratory burst oxidase RbohD, and the brassinosteroid receptor BRI1 (Bücherl et al. 2017; Hao et al. 2014; Wang et al. 2015). Membrane microdomains also play important roles in immune responses to pathogens. Rice plants with decreased amounts of sphingolipids and, thus, decreased abundance of DRM are more susceptible to M. oryzae (Nagano et al. 2016). In addition, full abundance of DRM is required for proper localization of the Rac/ROP small GTPase Rac1 and respiratory burst oxidase homologs $\mathrm{RbohB} / \mathrm{H}$ complex and for the chitin-induced ROS burst (Nagano et al. 2016). In humans, PRR such as Toll-like receptors cluster in specific membrane microdomains and this is critical for pathogen detection and activation of antifungal innate immunity (Inoue and Shinohara 2014; Ruysschaert and Lonez 2015). Thus, membrane microdomains are emerging as dynamic defense platforms that facilitate clustering of immune signaling complexes involved in pathogen detection and downstream signaling.

Quantitative mass spectrometry analyses of Arabidopsis DRM during cold acclimation and flagellin perception have identified several RIN4-associated proteins (Keinath et al. 2010; Minami et al. 2009) (Fig. 2). Plasma membrane $\mathrm{H}^{+}$ -ATPases, including AHA1 and AHA2, have been consistently found in DRM of Arabidopsis and N. tabacum (Keinath et al. 2010; Minami et al. 2009; Morel et al. 2006). FLS2 and nine other receptor-like kinases are among the most enriched proteins in DRM after flg22 elicitation (Keinath et al. 2010). This indicates dynamic partitioning of PRR to DRM after pathogen perception and highlights the importance of membrane microdomains as platforms for immune signaling. Further evidence for the presence of RIN4-associated proteins in DRM comes from the association of the RPS2 NLR with two DRM-enriched hypersensitive induced reaction proteins (AtHIR1 and AtHIR2) that contribute to RPS2-mediated NTI (Qi et al. 2011). AtHIR proteins contain the stomatin/prohibitin/flotillin/HflK/C (SPFH) domain, present in membrane proteins that localize to membrane microdomains (Browman et al. 2007; Qi et al. 2011). Enrichment of RIN4-associated, immune regulatory proteins to DRM indicates that RIN4 may function as a hub in plant immunity by regulating dynamic protein complex formation within membrane microdomains. Interestingly, while activated FLS2 increases in abundance in DRM, FLS2 is also subjected to clathrin-mediated endocytosis after activation, which occurs at sites independent of DRM (Robatzek et al. 2006). Given the role of RIN4 in regulating FLS2- and other DRM-localized, defense-signaling complexes, it is possible that RIN4, dependent on its PTM, acts to partition FLS2 and other defense signaling proteins to distinct membrane locales.

\section{CONCLUSIONS}

Based on the intrinsically disordered structure of RIN4, which facilitates its ability to associate with different client proteins, and its known associations with immune regulatory proteins localized to DRM, we propose that RIN4 functions as a scaffold for dynamic assembly of immune regulatory protein complexes at discrete membrane microdomains, possibly with effector- or host-induced PTM driving its domain switching. For many RIN4-associated proteins, it remains to be elucidated whether the in-planta association is the result of direct protein- 
protein interactions or indirect protein associations within putative membrane microdomains. Although association of immune regulatory proteins at the PM has been intensely studied, the role of protein-lipid interactions in bridging protein complex formation is under-explored. As such, further study of RIN4 will reveal how membrane partitioning contributes to the dynamic formation of protein complexes with various roles in plant immunity.

RIN4 PTM at specific residues are differentially involved in activation or suppression of immune responses, indicating that PTM act as molecular switches for RIN4 function. Importantly, modified residues and regions targeted by PTM are largely conserved across RIN4 homologs. Thus, using genome editing technology to target sites of PTM in RIN4 homologs could be employed to boost disease resistance in crop plants. Furthermore, more comprehensive analyses of PTM of RIN4 and other NOI domain-containing proteins and their respective roles in response to diverse biotic and abiotic stresses may reveal additional residues that could be precisely targeted for crop improvement.

\section{LITERATURE CITED}

Aarts, N., Metz, M., Holub, E., Staskawicz, B. J., Daniels, M. J., and Parker, J. E. 1998. Different requirements for EDS1 and NDR1 by disease resistance genes define at least two $R$ gene-mediated signaling pathways in Arabidopsis. Proc. Natl. Acad. Sci. U.S.A. 95:10306-10311.

Afzal, A. J., da Cunha, L., and Mackey, D. 2011. Separable fragments and membrane tethering of Arabidopsis RIN4 regulate its suppression of PAMP-triggered immunity. Plant Cell 23:3798-3811.

Afzal, A. J., Kim, J. H., and Mackey, D. 2013. The role of NOI-domain containing proteins in plant immune signaling. BMC Genomics 14:327.

Ashfield, T., Keen, N. T., Buzzell, R. I., and Innes, R. W. 1995. Soybean resistance genes specific for different Pseudomonas syringae avirulence genes are allelic, or closely linked, at the RPG1 locus. Genetics 141: 1597-1604.

Ashfield, T., Ong, L. E., Nobuta, K., Schneider, C. M., and Innes, R. W. 2004. Convergent evolution of disease resistance gene specificity in two flowering plant families. Plant Cell 16:309-318.

Ashfield, T., Redditt, T., Russell, A., Kessens, R., Rodibaugh, N., Galloway, L., Kang, Q., Podicheti, R., and Innes, R. W. 2014. Evolutionary relationship of disease resistance genes in soybean and Arabidopsis specific for the Pseudomonas syringae effectors AvrB and AvrRpm1. Plant Physiol. 166:235-251.

Assmann, S. M., Simoncini, L., and Schroeder, J. I. 1985. Blue light activates electrogenic ion pumping in guard cell protoplasts of Vicia faba. Nature 318:285-287.

Axtell, M. J., Chisholm, S. T., Dahlbeck, D., and Staskawicz, B. J. 2003. Genetic and molecular evidence that the Pseudomonas syringae type III effector protein AvrRpt2 is a cysteine protease. Mol. Microbiol. 49: 1537-1546.

Axtell, M. J., and Staskawicz, B. J. 2003. Initiation of RPS2-specified disease resistance in Arabidopsis is coupled to the AvrRpt2-directed elimination of RIN4. Cell 112:369-377.

Batistic, O. 2012. Genomics and localization of the Arabidopsis DHHCcysteine-rich domain $S$-acyltransferase protein family. Plant Physiol. 160:1597-1612.

Belkhadir, Y., Nimchuk, Z., Hubert, D. A., Mackey, D., and Dangl, J. L. 2004. Arabidopsis RIN4 negatively regulates disease resistance mediated by RPS2 and RPM1 downstream or independent of the NDR1 signal modulator and is not required for the virulence functions of bacterial type III effectors AvrRpt2 or AvrRpm1. Plant Cell 16:2822-2835.

Bisgrove, S. R., Simonich, M. T., Smith, N. M., Sattler, A., and Innes, R. W. 1994. A disease resistance gene in Arabidopsis with specificity for two different pathogen avirulence genes. Plant Cell 6:927-933.

Block, A., Li, G., Fu, Z. Q., and Alfano, J. R. 2008. Phytopathogen type III effector weaponry and their plant targets. Curr. Opin. Plant Biol. 11: 396-403.

Bomblies, K., Lempe, J., Epple, P., Warthmann, N., Lanz, C., Dangl, J. L., and Weigel, D. 2007. Autoimmune response as a mechanism for a Dobzhansky-Muller-type incompatibility syndrome in plants. PLoS Biol. 5:e236.

Boyes, D. C., Nam, J., and Dangl, J. L. 1998. The Arabidopsis thaliana $R P M 1$ disease resistance gene product is a peripheral plasma membrane protein that is degraded coincident with the hypersensitive response. Proc. Natl. Acad. Sci. U.S.A. 95:15849-15854.

Browman, D. T., Hoegg, M. B., and Robbins, S. M. 2007. The SPFH domain-containing proteins: More than lipid raft markers. Trends Cell Biol. 17:394-402.

Bücherl, C. A., Jarsch, I. K., Schudoma, C., Segonzac, C., Mbengue, M., Robatzek, S., MacLean, D., Ott, T., and Zipfel, C. 2017. Plant immune and growth receptors share common signalling components but localise to distinct plasma membrane nanodomains. eLife 6:e25114.

Cherkis, K. A., Temple, B. R. S., Chung, E. H., Sondek, J., and Dangl, J. L. 2012. AvrRpm1 missense mutations weakly activate RPS2-mediated immune response in Arabidopsis thaliana. PLoS One 7:e42633.

Chiang, Y.-H., and Coaker, G. 2015. Effector-triggered immunity: NLR immune perception and downstream defense responses. Page e0183 in: The Arabidopsis Book. The American Society of Plant Biologists, Rockville, MD, U.S.A

Chisholm, S. T., Dahlbeck, D., Krishnamurthy, N., Day, B., Sjolander, K., and Staskawicz, B. J. 2005. Molecular characterization of proteolytic cleavage sites of the Pseudomonas syringae effector AvrRpt2. Proc. Natl. Acad. Sci. U.S.A. 102:2087-2092.

Chung, E. H., da Cunha, L., Wu, A. J., Gao, Z., Cherkis, K., Afzal, A. J., Mackey, D., and Dangl, J. L. 2011. Specific threonine phosphorylation of a host target by two unrelated type III effectors activates a host innate immune receptor in plants. Cell Host Microbe 9:125-136.

Chung, E. H., El-Kasmi, F., He, Y., Loehr, A., and Dangl, J. L. 2014. A plant phosphoswitch platform repeatedly targeted by type III effector proteins regulates the output of both tiers of plant immune receptors. Cell Host Microbe 16:484-494

Coaker, G., Falick, A., and Staskawicz, B. 2005. Activation of a phytopathogenic bacterial effector protein by a eukaryotic cyclophilin. Science 308:548-550.

Coppinger, P., Repetti, P. P., Day, B., Dahlbeck, D., Mehlert, A., and Staskawicz, B. J. 2004. Overexpression of the plasma membranelocalized NDR1 protein results in enhanced bacterial disease resistance in Arabidopsis thaliana. Plant J. 40:225-237.

Cunnac, S., Lindeberg, M., and Collmer, A. 2009. Pseudomonas syringae type III secretion system effectors: Repertoires in search of functions Curr. Opin. Microbiol. 12:53-60.

Day, B., Dahlbeck, D., Huang, J., Chisholm, S. T., Li, D., and Staskawicz, B. J. 2005. Molecular basis for the RIN4 negative regulation of RPS2 disease resistance. Plant Cell 17:1292-1305.

Day, B., Dahlbeck, D., and Staskawicz, B. J. 2006. NDR1 interaction with RIN4 mediates the differential activation of multiple disease resistance pathways in Arabidopsis. Plant Cell 18:2782-2791.

de Lucas, M., Davière, J. M., Rodríguez-Falcón, M., Pontin, M., IglesiasPedraz, J. M., Lorrain, S., Fankhauser, C., Blázquez, M. A., Titarenko, E., and Prat, S. 2008. A molecular framework for light and gibberellin control of cell elongation. Nature 451:480-484.

Deslandes, L., and Rivas, S. 2012. Catch me if you can: Bacterial effectors and plant targets. Trends Plant Sci. 17:644-655.

Dodds, P. N., and Rathjen, J. P. 2010. Plant immunity: Towards an integrated view of plant-pathogen interactions. Nat. Rev. Genet. 11: 539-548.

Drazic, A., Myklebust, L. M., Ree, R., and Arnesen, T. 2016. The world of protein acetylation. Biochim. Biophys. Acta 1864:1372-1401.

Elmore, J. M., and Coaker, G. 2011. The role of the plasma membrane $\mathrm{H}^{+}$-ATPase in plant-microbe interactions. Mol. Plant 4:416-427.

Feng, S., Martinez, C., Gusmaroli, G., Wang, Y., Zhou, J., Wang, F., Chen, L., Yu, L., Iglesias-Pedraz, J. M., Kircher, S., Schäfer, E., Fu, X., Fan, L. M., and Deng, X. W. 2008. Coordinated regulation of Arabidopsis thaliana development by light and gibberellins. Nature 451:475-479.

Fujisaki, K., Abe, Y., Ito, A., Saitoh, H., Yoshida, K., Kanzaki, H., Kanzaki, E., Utsushi, H., Yamashita, T., Kamoun, S., and Terauchi, R. 2015. Rice Exo70 interacts with a fungal effector, AVR-Pii, and is required for AVR-Pii-triggered immunity. Plant J. 83:875-887.

Grant, M. R., Godiard, L., Straube, E., Ashfield, T., Lewald, J., Sattler, A., Innes, R. W., and Dangl, J. L. 1995. Structure of the Arabidopsis RPM1 gene enabling dual specificity disease resistance. Science 269:843-846.

Hao, H., Fan, L., Chen, T., Li, R., Li, X., He, Q., Botella, M. A., and Lin, J. 2014. Clathrin and membrane microdomains cooperatively regulate RbohD dynamics and activity in Arabidopsis. Plant Cell 26:1729-1745.

Hemsley, P. A. 2015. The importance of lipid modified proteins in plants. New Phytol. 205:476-489.

Hou, X., Lee, L. Y., Xia, K., Yan, Y., and Yu, H. 2010. DELLAs modulate jasmonate signaling via competitive binding to JAZs. Dev. Cell 19: 884-894.

Hsu, W. L., Oldfield, C. J., Xue, B., Meng, J., Huang, F., Romero, P., Uversky, V. N., and Dunker, A. K. 2013. Exploring the binding diversity 
of intrinsically disordered proteins involved in one-to-many binding. Protein Sci. 22:258-273.

Iakoucheva, L. M., Brown, C. J., Lawson, J. D., Obradović, Z., and Dunker, A. K. 2002. Intrinsic disorder in cell-signaling and cancer-associated proteins. J. Mol. Biol. 323:573-584.

Inoue, M., and Shinohara, M. L. 2014. Clustering of pattern recognition receptors for fungal detection. PLoS Pathog. 10:e1003873.

Jeuken, M. J., Zhang, N. W., McHale, L. K., Pelgrom, K., den Boer, E., Lindhout, P., Michelmore, R. W., Visser, R. G., and Niks, R. E. 2009. Rin4 causes hybrid necrosis and race-specific resistance in an interspecific lettuce hybrid. Plant Cell 21:3368-3378.

Jin, P., Wood, M. D., Wu, Y., Xie, Z., and Katagiri, F. 2003. Cleavage of the Pseudomonas syringae type III effector AvrRpt2 requires a host factor(s) common among eukaryotes and is important for AvrRpt 2 localization in the host cell. Plant Physiol. 133:1072-1082.

Jones, J. D. G., and Dangl, J. L. 2006. The plant immune system. Nature 444:323-329.

Kaundal, A., Ramu, V. S., Oh, S., Lee, S., Pant, B., Lee, H. K., Rojas, C. M., Senthil-Kumar, M., and Mysore, K. S. 2017. GENERAL CONTROL NONREPRESSIBLE4 degrades 14-3-3 and the RIN4 complex to regulate stomatal aperture with implications on nonhost disease resistance and drought tolerance. Plant Cell 29:2233-2248.

Keinath, N. F., Kierszniowska, S., Lorek, J., Bourdais, G., Kessler, S. A., Shimosato-Asano, H., Grossniklaus, U., Schulze, W. X., Robatzek, S. and Panstruga, R. 2010. PAMP (pathogen-associated molecular pattern)induced changes in plasma membrane compartmentalization reveal novel components of plant immunity. J. Biol. Chem. 285:39140-39149.

Khan, M., Subramaniam, R., and Desveaux, D. 2016. Of guards, decoys, baits and traps: Pathogen perception in plants by type III effector sensors. Curr. Opin. Microbiol. 29:49-55.

Kim, H. S., Desveaux, D., Singer, A. U., Patel, P., Sondek, J., and Dangl, J. L. 2005a. The Pseudomonas syringae effector AvrRpt2 cleaves its Cterminally acylated target, RIN4, from Arabidopsis membranes to block RPM1 activation. Proc. Natl. Acad. Sci. U.S.A. 102:6496-6501.

Kim, M. G., da Cunha, L., McFall, A. J., Belkhadir, Y., DebRoy, S., Dangl, J. L., and Mackey, D. 2005b. Two Pseudomonas syringae type III effectors inhibit RIN4-regulated basal defense in Arabidopsis. Cell 121: 749-759.

Kim, T. H., Böhmer, M., Hu, H., Nishimura, N., and Schroeder, J. I. 2010. Guard cell signal transduction network: Advances in understanding abscisic acid, $\mathrm{CO}_{2}$, and $\mathrm{Ca}^{2+}$ signaling. Annu. Rev. Plant Biol. 61: 561-591.

Knepper, C., Savory, E. A., and Day, B. 2011. Arabidopsis NDR1 is an integrin-like protein with a role in fluid loss and plasma membrane-cell wall adhesion. Plant Physiol. 156:286-300.

Lee, D., Bourdais, G., Yu, G., Robatzek, S., and Coaker, G. 2015a. Phosphorylation of the plant immune regulator RPM1-INTERACTING PROTEIN4 enhances plant plasma membrane $\mathrm{H}^{+}$-ATPase activity and inhibits flagellin-triggered immune responses in Arabidopsis. Plant Cell 27:2042-2056.

Lee, J., Manning, A. J., Wolfgeher, D., Jelenska, J., Cavanaugh, K. A., Xu, H., Fernandez, S. M., Michelmore, R. W., Kron, S. J., and Greenberg, J. T. 2015b. Acetylation of an NB-LRR plant immune-effector complex suppresses immunity. Cell Reports 13:1670-1682.

Lewis, J. D., Lee, A., Ma, W., Zhou, H., Guttman, D. S., and Desveaux, D. 2011. The YopJ superfamily in plant-associated bacteria. Mol. Plant Pathol. 12:928-937.

Li, L., Li, M., Yu, L., Zhou, Z., Liang, X., Liu, Z., Cai, G., Gao, L., Zhang, X., Wang, Y., Chen, S., and Zhou, J. M. 2014b. The FLS2-associated kinase BIK1 directly phosphorylates the NADPH oxidase RbohD to control plant immunity. Cell Host Microbe 15:329-338.

Li, M., Ma, X., Chiang, Y. H., Yadeta, K. A., Ding, P., Dong, L., Zhao, Y., Li, X., Yu, Y., Zhang, L., Shen, Q. H., Xia, B., Coaker, G., Liu, D., and Zhou, J. M. 2014a. Proline isomerization of the immune receptorinteracting protein RIN4 by a cyclophilin inhibits effector-triggered immunity in Arabidopsis. Cell Host Microbe 16:473-483.

Lim, M. T., and Kunkel, B. N. 2004. Mutations in the Pseudomonas syringae avrRpt2 gene that dissociate its virulence and avirulence activities lead to decreased efficiency in AvrRpt2-induced disappearance of RIN4. Mol. Plant-Microbe Interact. 17:313-321.

Liu, J., Elmore, J. M., Fuglsang, A. T., Palmgren, M. G., Staskawicz, B. J., and Coaker, G. 2009. RIN4 functions with plasma membrane $\mathrm{H}^{+}$-ATPases to regulate stomatal apertures during pathogen attack. PLoS Biol. 7:e1000139.

Liu, J., Elmore, J. M., Lin, Z. J., and Coaker, G. 2011. A receptor-like cytoplasmic kinase phosphorylates the host target RIN4, leading to the activation of a plant innate immune receptor. Cell Host Microbe 9: 137-146.
Lu, D., Wu, S., Gao, X., Zhang, Y., Shan, L., and He, P. 2010. A receptorlike cytoplasmic kinase, BIK1, associates with a flagellin receptor complex to initiate plant innate immunity. Proc. Natl. Acad. Sci. U.S.A. 107:496-501.

Luo, Y., Caldwell, K. S., Wroblewski, T., Wright, M. E., and Michelmore, R. W. 2009. Proteolysis of a negative regulator of innate immunity is dependent on resistance genes in tomato and Nicotiana benthamiana and induced by multiple bacterial effectors. Plant Cell 21:2458-2472.

Mackey, D., Belkhadir, Y., Alonso, J. M., Ecker, J. R., and Dangl, J. L. 2003. Arabidopsis RIN4 is a target of the type III virulence effector AvrRpt2 and modulates RPS2-mediated resistance. Cell 112:379-389.

Mackey, D., Holt, B. F., 3rd, Wiig, A., and Dangl, J. L. 2002. RIN4 interacts with Pseudomonas syringae type III effector molecules and is required for RPM1-mediated resistance in Arabidopsis. Cell 108:743-754.

Malinsky, J., Opekarová, M., Grossmann, G., and Tanner, W. 2013. Membrane microdomains, rafts, and detergent-resistant membranes in plants and fungi. Annu. Rev. Plant Biol. 64:501-529.

Melotto, M., Underwood, W., Koczan, J., Nomura, K., and He, S. Y. 2006. Plant stomata function in innate immunity against bacterial invasion. Cell 126:969-980.

Minami, A., Fujiwara, M., Furuto, A., Fukao, Y., Yamashita, T., Kamo, M., Kawamura, Y., and Uemura, M. 2009. Alterations in detergent-resistant plasma membrane microdomains in Arabidopsis thaliana during cold acclimation. Plant Cell Physiol. 50:341-359.

Mohan, A., Oldfield, C. J., Radivojac, P., Vacic, V., Cortese, M. S., Dunker, A. K., and Uversky, V. N. 2006. Analysis of molecular recognition features (MoRFs). J. Mol. Biol. 362:1043-1059.

Morel, J., Claverol, S., Mongrand, S., Furt, F., Fromentin, J., Bessoule, J. J., Blein, J. P., and Simon-Plas, F. 2006. Proteomics of plant detergentresistant membranes. Mol. Cell. Proteomics 5:1396-1411.

Mukherjee, S., Keitany, G., Li, Y., Wang, Y., Ball, H. L., Goldsmith, E. J., and Orth, K. 2006. Yersinia YopJ acetylates and inhibits kinase activation by blocking phosphorylation. Science 312:1211-1214.

Nagano, M., Ishikawa, T., Fujiwara, M., Fukao, Y., Kawano, Y., KawaiYamada, M., and Shimamoto, K. 2016. Plasma membrane microdomains are essential for Rac1-RbohB/H-mediated immunity in rice. Plant Cell 28:1966-1983.

Nimchuk, Z., Marois, E., Kjemtrup, S., Leister, R. T., Katagiri, F., and Dangl, J. L. 2000. Eukaryotic fatty acylation drives plasma membrane targeting and enhances function of several type III effector proteins from Pseudomonas syringae. Cell 101:353-363.

Oldfield, C. J., Cheng, Y., Cortese, M. S., Brown, C. J., Uversky, V. N., and Dunker, A. K. 2005a. Comparing and combining predictors of mostly disordered proteins. Biochemistry 44:1989-2000.

Oldfield, C. J., Cheng, Y., Cortese, M. S., Romero, P., Uversky, V. N., and Dunker, A. K. 2005b. Coupled folding and binding with alpha-helixforming molecular recognition elements. Biochemistry 44:12454-12470.

Oldfield, C. J., and Dunker, A. K. 2014. Intrinsically disordered proteins and intrinsically disordered protein regions. Annu. Rev. Biochem. 83: 553-584.

Palazzo, L., Mikoč, A., and Ahel, I. 2017. ADP-ribosylation: New facets of an ancient modification. FEBS J. 284:2932-2946.

Partch, C. L., Clarkson, M. W., Ozgür, S., Lee, A. L., and Sancar, A. 2005. Role of structural plasticity in signal transduction by the cryptochrome blue-light photoreceptor. Biochemistry 44:3795-3805.

Pedley, K. F., and Martin, G. B. 2003. Molecular basis of Pto-mediated resistance to bacterial speck disease in tomato. Annu. Rev. Phytopathol. 41:215-243.

Qi, Y., and Katagiri, F. 2012. Membrane microdomain may be a platform for immune signaling. Plant Signal. Behav. 7:454-456.

Qi, Y., Tsuda, K., Nguyen, V., Wang, X., Lin, J., Murphy, A. S., Glazebrook, J., Thordal-Christensen, H., and Katagiri, F. 2011. Physical association of Arabidopsis hypersensitive induced reaction proteins (HIRs) with the immune receptor RPS2. J. Biol. Chem. 286:31297-31307.

Ritter, C., and Dangl, J. L. 1996. Interference between two specific pathogen recognition events mediated by distinct plant disease resistance genes. Plant Cell 8:251-257.

Robatzek, S., Chinchilla, D., and Boller, T. 2006. Ligand-induced endocytosis of the pattern recognition receptor FLS2 in Arabidopsis. Genes Dev. 20:537-542.

Robert-Seilaniantz, A., Shan, L., Zhou, J.-M., and Tang, X. 2006. The Pseudomonas syringae pv. tomato DC3000 type III effector HopF2 has a putative myristoylation site required for its avirulence and virulence functions. Mol. Plant-Microbe Interact. 19:130-138.

Ruysschaert, J. M., and Lonez, C. 2015. Role of lipid microdomains in TLR-mediated signalling. Biochim. Biophys. Acta 1848:1860-1867.

Sabol, P., Kulich, I., and Žárský, V. 2017. RIN4 recruits the exocyst subunit EXO70B1 to the plasma membrane. J. Exp. Bot. 68:3253-3265. 
Selote, D., and Kachroo, A. 2010. RPG1-B-derived resistance to AvrBexpressing Pseudomonas syringae requires RIN4-like proteins in soybean. Plant Physiol. 153:1199-1211.

Selote, D., Robin, G. P., and Kachroo, A. 2013. GmRIN4 protein family members function nonredundantly in soybean race-specific resistance against Pseudomonas syringae. New Phytol. 197:1225-1235.

Shan, L., Thara, V. K., Martin, G. B., Zhou, J. M., and Tang, X. 2000. The Pseudomonas AvrPto protein is differentially recognized by tomato and tobacco and is localized to the plant plasma membrane. Plant Cell 12: 2323-2338.

Shimazaki, K., Lino, M., and Zeiger, E. 1986. Blue light-dependent proton extrusion by guard-cell protoplasts of Vicia faba. Nature 319: 324-326.

Shultz, J. L., Kurunam, D., Shopinski, K., Iqbal, M. J., Kazi, S., Zobrist, K., Bashir, R., Yaegashi, S., Lavu, N., Afzal, A. J., Yesudas, C. R., Kassem, M. A., Wu, C., Zhang, H. B., Town, C. D., Meksem, K., and Lightfoot, D. A. 2006. The Soybean Genome Database (SoyGD): A browser for display of duplicated, polyploid, regions and sequence tagged sites on the integrated physical and genetic maps of Glycine max. Nucleic Acids Res. 34:D758-D765.

Sun, T. P. 2011. The molecular mechanism and evolution of the GA-GID1DELLA signaling module in plants. Curr. Biol. 21:R338-R345.

Sun, X., Greenwood, D. R., Templeton, M. D., Libich, D. S., McGhie, T. K., Xue, B., Yoon, M., Cui, W., Kirk, C. A., Jones, W. T., Uversky, V. N., and Rikkerink, E. H. 2014. The intrinsically disordered structural platform of the plant defence hub protein RPM1-interacting protein 4 provides insights into its mode of action in the host-pathogen interface and evolution of the nitrate-induced domain protein family. FEBS J. 281: 3955-3979.

Sun, X., Jones, W. T., and Rikkerink, E. H. 2012. GRAS proteins: The versatile roles of intrinsically disordered proteins in plant signalling. Biochem. J. 442:1-12.

Sun, X., Rikkerink, E. H., Jones, W. T., and Uversky, V. N. 2013. Multifarious roles of intrinsic disorder in proteins illustrate its broad impact on plant biology. Plant Cell 25:38-55.

Takemoto, D., and Jones, D. A. 2005. Membrane release and destabilization of Arabidopsis RIN4 following cleavage by Pseudomonas syringae AvrRpt2. Mol. Plant-Microbe Interact. 18:1258-1268.

Thomma, B. P. H. J., Nürnberger, T., and Joosten, M. H. A. J. 2011. Of PAMPs and effectors: The blurred PTI-ETI dichotomy. Plant Cell 23: 4-15.
Toruño, T. Y., Stergiopoulos, I., and Coaker, G. 2016. Plant-pathogen effectors: Cellular probes interfering with plant defenses in spatial and temporal manners. Annu. Rev. Phytopathol. 54:419-441.

Wang, L., Li, H., Lv, X., Chen, T., Li, R., Xue, Y., Jiang, J., Jin, B., Baluška, F., Šamaj, J., Wang, X., and Lin, J. 2015. Spatiotemporal dynamics of the BRI1 receptor and its regulation by membrane microdomains in living Arabidopsis cells. Mol. Plant 8:1334-1349.

Wang, Y., Li, J., Hou, S., Wang, X., Li, Y., Ren, D., Chen, S., Tang, X., and Zhou, J. M. 2010. A Pseudomonas syringae ADP-ribosyltransferase inhibits Arabidopsis mitogen-activated protein kinase kinases. Plant Cell 22:2033-2044.

Wild, M., Davière, J. M., Cheminant, S., Regnault, T., Baumberger, N., Heintz, D., Baltz, R., Genschik, P., and Achard, P. 2012. The Arabidopsis DELLA RGA-LIKE3 is a direct target of MYC2 and modulates jasmonate signaling responses. Plant Cell 24:3307-3319.

Wilton, M., Subramaniam, R., Elmore, J., Felsensteiner, C., Coaker, G., and Desveaux, D. 2010. The type III effector HopF $2_{\text {Pto }}$ targets Arabidopsis RIN4 protein to promote Pseudomonas syringae virulence. Proc. Natl. Acad. Sci. U.S.A. 107:2349-2354.

Wright, P. E., and Dyson, H. J. 2015. Intrinsically disordered proteins in cellular signalling and regulation. Nat. Rev. Mol. Cell Biol. 16:18-29.

Xu, N., Luo, X., Li, W., Wang, Z., and Liu, J. 2017. The bacterial effector AvrB-induced RIN4 hyperphosphorylation is mediated by a receptorlike cytoplasmic kinase complex in Arabidopsis. Mol. Plant-Microbe Interact. 30:502-512.

Yoon, M. K., Shin, J., Choi, G., and Choi, B. S. 2006. Intrinsically unstructured N-terminal domain of bZIP transcription factor HY5. Proteins 65:856-866.

Zhang, J., Li, W., Xiang, T., Liu, Z., Laluk, K., Ding, X., Zou, Y., Gao, M., Zhang, X., Chen, S., Mengiste, T., Zhang, Y., and Zhou, J. M. 2010 Receptor-like cytoplasmic kinases integrate signaling from multiple plant immune receptors and are targeted by a Pseudomonas syringae effector. Cell Host Microbe 7:290-301.

Zipfel, C. 2014. Plant pattern-recognition receptors. Trends Immunol. 35: 345-351.

\section{AUTHOR-RECOMMENDED INTERNET RESOURCE}

Protein disorder prediction system (PrDOS) server: http://prdos.hgc.jp/cgi-bin/top.cgi 